\title{
UTILIZAÇÕES DA WISC-III NA AVALIAÇÃO NEUROPSICOLÓGICA DE CRIANÇAS E ADOLESCENTES ${ }^{1}$
}

\author{
Mário R. Simões ${ }^{2}$ \\ Universidade de Coimbra - Portugal
}

\begin{abstract}
RESUMO: Este texto pretende evidenciar os principais contributos da Terceira Edição da Escala de Inteligência de Wechsler (WISC-III) para a avaliação neuropsicológica de crianças e adolescentes. Alguns indicadores são objeto de exploração: Quociente Intelectual da Escala Completa, Quociente Intelectual verbal, Quociente Intelectual de realização, discrepância(s) entre QIv e QIr, Índices Factoriais, análise por subteste, variabilidade inter-testes, formas reduzidas, avaliação do nível de funcionamento cognitivo pré-mórbido, testes neuropsicológicos e funções psicológicas examinadas a partir da WISC-III. Neste contexto, são revistos alguns dos principais estudos com grupos clínicos de crianças com lesão cerebral traumática, cancro, espinha bífida, hidrocefalia, epilepsia. É feita uma referência breve ao futuro próximo da investigação relativa à utilização neuropsicológica da WISC-III.
\end{abstract}

Palavras-chave: WISC-III, avaliação neuropsicológica, testes neuropsicológicos, investigação, grupos clínicos.

\section{USES OF THE WISC-III IN THE ASSESSMENT NEUROPSYCHOLOGICAL OF CHILDREN IT IS ADOLESCENT}

\begin{abstract}
The present paper aims to explore the main clinical contributions of the Wechsler Intelligence Scale for Children-Third Edition (WISC-III) of child and adolescent neuropsychological assessment. Some of the main studies about WISC-III uses on neuropsychological assessment are reviewed. This analysis implies some references about Full Scale IQ, verbal and performance IQ and Index scores discrepancies, profile analysis, short forms, relationships between WISC-III and neuropsychological tests, premorbid prediction strategies. Some research data regarding patterns of cognitive functioning and the sensivity of the WISC-III among children with clinical problems like traumatic head injury, cancer, spina bifida, hydrocephalus and epilepsy are also presented. Finally, a brief reference is made on some trends for future research with neuropsychological WISC-III usage.
\end{abstract}

Key-words: WISC-III, neuropsychological assessment, neuropsychological tests, research, clinical groups.

Um dos primeiros e principais objetivos da avaliação neuropsicológica foi o de identificar sujeitos com lesão cerebral distinguindo-os dos sujeitos normais, ou localizar lesões estruturais cuja existência se suspeitava a partir da interpretação dos padrões de desempenho numa prova constituída por testes diversificados (como as Escalas de Inteligência de Wechsler). Ainda numa perspectiva histórica

\footnotetext{
${ }^{1}$ Artigo recebido para publicação em março de 2002; aceito em maio de 2002

2 Serviço de Avaliação Psicológica. Faculdade de Psicologia e de Ciências da Educação da Universidade de Coimbra. Rua do Colégio Novo, 3001-802 Coimbra (Portugal), E-mail: simoesmr@hotmail.com
}

Baron (2001) recorda que a avaliaçã̃o neuropsicológica foi freqüentemente considerada como concluída sempre que incluísse uma medida de inteligência geral (QI), uma prova de realização escolar, um teste de despiste da linguagem e, mais ocasionalmente, um exame rápido da motricidade $\mathrm{e}$ das funções perceptivo-sensoriais.

A Escala de Inteligência de Wechsler para Crianças - Terceira Edição (WISC-III; Wechsler, 1991) é nos nossos dias a principal referência, a melhor e a mais utilizada medida da inteligência de crianças e adolescentes, encontrando-se agora em fase de aferição em países como o Brasil (cf. 


\section{Mário R. Simões}

Figueiredo, 2000) e Portugal.

Além disso, trata-se de um instrumenta sempre presente na avaliação neuropsicológica. A sua aplicação constitui parte integrante na avaliação neuropsicológica compreensiva de crianças e adolescentes (cf., por exemplo, Anderson, Northam, Hendy \& Wrennall, 2001; Groth-Marnat, Gallagher, Hale \& Kaplan, 2000; Lezak, 1995; Riccio \& Reynolds, 1998; Spreen \& Strauss, 1998).

Neste plano Manga e Fournier (1997) advertem que a WISC-III foi construída como um instrumento de avaliação da inteligência e não como uma medida do funcionamento neurológico. No entanto, e apesar de não ser propriamente uma prova neuropsicológica, a WISC-III é amplamente utilizada em neuropsicologia e encontra-se mesmo entre os instrumentos mais freqüentemente usados em avaliação neuropsicológica (Horowitz, Schatz \& Chute, 1997) sendo incluída na maioria das avaliações neuropsicológicas pediátricas (Donders \& Warschausky, 1996a). Este fato sugere que se trata de uma prova útil e essencial no processo de avaliação neuropsicológica.

Toda a avaliação neuropsicológica deve comportar um exame das funções intelectuais verbais e não verbais. $O$ ideal é mesmo começar a avaliação neuropsicológica com o exame da inteligência através da utilização de uma prova de referência como é o caso da WISC-III. Esta prova serve para estabelecer um padrão de comparação intra e interindividual e permite identificar um nível global de aptidão cognitiva (ou uma estimativa do potencial intelectual) e, assim, verificar se um desempenho pobre num teste sugere a presença de um déficit cognitivo específico ou, pelo contrário, constitui evidência de déficit intelectual generalizado.

Através dos seus subtestes a WISC-III viabiliza uma primeira distinção entre aptidões ou funções psicológicas, proporcionando um ponto de referência para o exame das funções corticais superiores. Neste contexto, a WISC-III serve para orientar as hipóteses sobre as áreas de disfuncionamento cognitivo e para a escolha das provas complementares, com o objetivo de alcançar um diagnóstico diferencial. Nas palavras de Riccio \& Reynolds (1998) o recurso inicial a uma prova de avaliação da inteligência como a WISC-III é essencial para fornecer uma linha-de-base para interpretar todas as outras funções.

A seguir à avaliação do funcionamento intelectual global através da WISC-III é geralmente necessário considerar também outras funções neuropsicológicas e perspectivar a utilização de provas neuropsicológicas mais específicas. No modelo do teste de hipóteses (Anderson \& Gilandis, 1994; Saling, 1994; Walsh, 1994), os testes selecionados para um segundo momento de avaliação devem ter em consideração as áreas cognitivas fortes e fracas evidenciadas pela criança na WISC-III e o modo como o perfil de aptidão pode interagir com as exigências colocadas à criança em casa e na escola. Riccio e Reynolds (1998) defendem que devem ser avaliadas todas as aptidões, ou pelo menos a maior parte das aptidões cognitivas e das aptidões de processamento de informação de ordem mais elevada, relevantes para a escolarização da criança.

Todavia, isoladamente, $o$ exame da inteligência não responde a muitas das necessidades da avaliação neuropsicológica, uma vez que, como referem Anderson, Northam, Hendy e Wrennall (2001), a identificação do nível intelectual não proporciona informações relativamente a muitos aspectos do funcionamento que são relevantes para a função do Sistema Nervoso Central (SNC). Além disso, o QI observado em crianças com várias perturbações do SNC encontra-se freqüentemente dentro da amplitude média. Isso significa que os testes de QI podem constituir um indicador insensível a várias disfunções neuropsicológicas comuns e insidiosas justificando o recurso inevitável a testes neuropsicológicos mais específicos.

Que interpretações acerca do funcionamento neuropsicológico podem então ser viabilizadas a partir da aplicação de provas de inteligência como a WISC-III e qual o seu grau de utilidade? O propósito do presente texto é justamente o de analisar os contributos e as circunstâncias de utilização da WISC-III no contexto da avaliação e investigação neuropsicológicas.

Uma forma de responder a este objetivo pode passar pela análise dos principais indicadores e potencialidades que esta escala de inteligência proporciona: QIEC (Quociente Intelectual da Escala Completa), QIv (Quociente Intelectual verbal), QIr 
(Quociente Intelectual de realização), diferenças entre QIv e QIr, Índices Fatoriais, resultados em subtestes isolados, recurso a Formas Reduzidas, estudos com grupos clínicos específicos.

\section{Quociente Intelectual}

Convém começar por referir um dos significados neuropsicológicos da avaliação da capacidade intelectual através de uma escala de inteligência como a WISC-III. Na investigação em neuropsicologia, a identificação preliminar do QI funda uma das precauções metodológicas atualmente mais valorizadas na constituição de grupos clíni$\cos$. O exame normativo de funções como a linguagem, a memória ou a atenção requer, freqüentemente, o controlo da variável QI, sendo habitualmente exigido um QIEC > 85 (ou, mais raramente QIEC $>80$ ) e a exclusão de psicopatologia (ansiedade, depressão, distúrbio da conduta), perturbações neurológicas (como epilepsia, tumores cerebrais, síndroma Gilles de La Tourette, neurofibromatose ou paralisia cerebral) ou dificuldades de aprendizagem.

A avaliação neuropsicológica, implementada através do recurso a provas mais específicas, requer habitualmente a observância prévia dos mesmos critérios mínimos de QI. A indicação do QI é necessária para um diagnóstico rigoroso, para o prognóstico e a reabilitação vocacional.

No entanto, $O$ QI pode não ser o indicador mais útil numa interpretação clínica específica. Neste plano, o interesse da WISC-III assenta, igualmente, na possibilidade de comparação entre o QIv e o QIr. Fletche, Levin e Butler (1995) referem que muitos estudos comprovam a este respeito que nos casos de lesão cerebral traumática é mais provável encontrar um decréscimo nas medidas de inteligência não verbais do que nas tarefas verbais. Outros parâmetros de interesse na WISC-III radicam na obtenção de um padrão de resultados individuais em vários subtestes, na conseqüente identificação de um perfil cognitivo e na sua correlação com problemas neurológicos, sem esquecer a análise de características qualitativas do desempenho. Variações nos resultados dos testes podem ser atribuídas a variáveis que não o verdadeiro nível de aptidão subjacente.

\section{QIv, QIr, discrepância(s) entre QIv e QIr}

Há investigação que relaciona o desempenho na WISC-III com a localização da disfunção cerebral. Alguma evidência de natureza correlacional, não conclusiva, oferece suporte para a idéia do QIv poder refletir o funcionamento do hemisfério esquerdo, ao mesmo tempo que o QIr traduziria a atividade do hemisfério direito. Estas hipóteses baseiam-se na distinção entre competências verbais e não-verbais, que é histórica e empiricamente evidenciada através de estudos de análise fatorial e que continua a ser aplicável à WISC-III. Neste contexto, do ponto de vista histórico, e de um modo particular em crianças, diferenças importantes entre QIv e QIr nas Escalas de Inteligência de Wechsler, têm sido usadas como um marcador de uma especialização hemisférica ou de déficits de processamento definidos de modo amplo, disfunção cerebral lateralizada e dificuldades da aprendizagem.

Manga e Fournier (1997) referem que nas crianças a alteração do sistema nervoso se traduz num desenvolvimento deficiente das capacidades cognitivas, podendo acarretar mais déficits no campo perceptivo-espacial ou no lingüístico. De um ponto de vista neurológico, a WISC não tem servido para estabelecer uma conexão sólida entre pontuações na subescala verbal com funções associadas ao hemisfério esquerdo, nem entre resultados na subescala de realização com funções residentes do hemisfério direito. Mais, a relação entre resultados nas Escalas de Inteligência de Wechsler e as funções hemisféricas cerebrais é mais frágil nas crianças (WISC) do que nos adultos (WAIS). A lesão sofrida por um adulto no hemisfério esquerdo pode afetar mais o QIv, do que o QIr, sendo opostos os resultados para a lesão no hemisfério direito. Manga e Fournier sublinham que o desenvolvimento e plasticidade cerebrais não permitem que se observem nas crianças relações semelhantes às encontradas com os adultos. Citando uma investigação de Riva e Cazzaniga (1986) aqueles autores lembram que quando se estudou o desempenho na WISC de crianças vítimas de lesões cerebrais unilaterais, antes ou depois de completarem um ano de vida, os resultados indicaram que as lesões no hemisfério esquerdo afetavam tanto o QIv 


\section{Mário R. Simões}

como $\circ$ QIr, independentemente do fato da lesão ter sido antes ou depois de um ano de idade; no entanto, os efeitos revelaram-se mais negativos nos casos em que a lesão ocorreu mais precocemente. Por outro lado, e também independentemente da lesão ser anterior ou posterior a um ano de vida, a lesão no hemisfério direito afetava apenas o QIr.

A parte manipulativa da escala (QIr) parece ser especialmente sensível à existência de uma lesão cerebral, de tal modo que as lesões ocorridas na infância tendem a mostrar um padrão QIv > QIr, "que parece associado à maior sensibilidade das tarefas de manipulação da WISC-R à alteração cerebral, mais do que à localização da lesão cerebral" (Telzrow, 1989 , p. 234). Num estudo longitudinal realizado por Moffitt e Silva (1987), apenas em 23\% dos casos com discrepância de 15 ou mais pontos se mantinha essa disparidade em dois ou mais níveis etários e, em geral, a diferença não estava associada nem a perturbaçōes de tipo neurológico, nem a outros problemas de comportamento ou de aprendizagem escolar (ainda que os casos em que o sentido da relação era QIv < QIr tenham mostrado rendimento acadêmico inferior ao das crianças com QIv > QIr).

Embora um maior número de sujeitos com djslexia apresente QIr > QIv a um maior número de sujeitos de um grupo de controlo e sujeitos com distúrbio de hiperatividade com déficits de atenção apresente um padrão QIv > QIr não foi identificado um padrão significativo de assimetria entre QIv e QIr. Apesar da discrepância QIr > QIv estar positivamente correlacionada com um melhor desempenho em testes de integração viso-espacial do que em testes de linguagem, o padrão QIv > QIr não está associado de modo consistente a um melhor desempenho em testes de linguagem comparativamente à realização em testes de integração viso-motora (Nielsen, Hynd \& Hiemenz, 1998).

Tem sido sugerido que uma discrepância significativa entre o QIv e o QIr (QIr > QIv) pode significar um quadro de dificuldades de aprendizagem $\mathrm{e} /$ ou a presença de um déficit da linguagem (Kamphaus, 1993). Em contraste, outros investigadores não consideram útil a utilização dos padrões de discrepância entre QIs, na diferenciação de crianças com dificuldades de aprendizagem (Humphries \& Bone, 1993). Slate (1995), num estudo com três grupos de crianças (dificuldades de aprendizagem, deficiência mental e outro tipo de diagnósticos) concluiu que todos os grupos apresentavam um QIr superior ao QIv na WISC-III. No entanto, a discrepância entre QIv e QIr foi mais pequena nas crianças deste estudo do que a encontrada na amostra normativa. Num estudo com crianças com problemas na área da linguagem, as discrepâncias entre QIv e QIr na WISC-III também foram pequenas (Phelps, Legouri, Nisewaner, \& Parker, 1993).

Ou seja, a investigação não tem proporcionado o necessário apoio da validade das discrepâncias QIv-QIr para fins de classificação ou de diagnóstico clínico. Divergências específicas entre QIv e QIr na WISC-III não tem qualquer valor (Kamphaus, 1993) e pode originar diagnósticos ou classificações erróneas (Prifitera \& Dersh, 1993). A investigação sugere que esta associação não é clinicamente fiável (Lezak, 1998). São pois necessárias precauções na interpretação dessas discrepâncias. Por exemplo, atribuir uma confiança excessiva ao valor da discrepância QIr > QIv como marcador das dificuldades de aprendizagem pode acarretar um diagnóstico incorreto (e intervenções inapropriadas) ou conduzir à ausência de formulação diagnóstica (e à não prestação de serviços de apoio especializado a crianças que apresentam dificuldades escolares). O mesmo se poderá dizer, aliás, em relação a outras condições clínicas. Quer isto dizer que a interpretação neuropsicológica deve ser crítica e apoiar-se nas observações feitas ao longo da administração das provas. Um desvio de 15 pontos a favor QIr não assinala obrigatoriamente um quadro de disfasia, ao mesmo tempo que uma discrepância de valor idêntico a favor do QIv não implica um perfil de dispraxia. A consideração dos resultados quantitativos jamais deverá obscurecer as observações qualitativas (Lussier \& Flessas, 2001). A exploração do significado estatístico para a diferença entre o QIv e o QI, ainda que possa sugerir uma lesão cerebral ou outro tipo de perturbação neurológica, deve ser desencorajada na prática de clínica infantil (Franzen, 2000; Reynolds, 1997a). No mesmo sentido, Kaufman (1994) aconselha que se tenha muita cautela no processo de interpretação diagnóstica, inclusive no caso de discrepâncias muito elevadas, uma vez que numa percentagem elevada de crianças nor- 
mais se observa uma diferença de 15 ou mais pontos entre QIv e QIr.

$\mathrm{Na}$ interpretação da diferença entre QIv e QIr deve ser considerada uma série de fatores. Esta inclui a presença de problemas ao nível da linguagem, audição, motricidade, motivação para a realização e diferenças lingüísticas ou culturais. Assim, apesar da discrepância QIv-QIr poder ser utilizada para formular hipóteses, a sua presença ou ausência não pode ser encarada como evidência conclusiva de uma incapacidade (Hynd, Cohen, Riccio \& Arceneaux, 1998).

Os limites da WISC-III (e da discrepância QIvQIr) para o diagnóstico neuropsicológico devem ser distinguidos das suas potencialidades ao nível da caracterização cognitiva. Com freqüência a dicotomia QIv - QIr parece revelar-se útil para saber se a criança apresenta um déficit que apenas afeta as capacidades lingüísticas (QIv), ou as capacidades perceptivo-espaciais (QIr). Pela análise do predomínio relativo destas competências pode saber-se se as áreas fracas da capacidade intelectual da criança correspondem às suas competências lingüísticas (QIv $<$ QIr), ou às competências perceptivo-espaciais ( $\mathrm{QIr}$ $<$ QIv). Podemos dizer que uma discrepância significativa entre estes dois QIs tem uma possível origem neurológica caso, juntamente com os restantes resultados de uma avaliação neuropsicológica suficientemente ampla e apropriada, contribua para um melhor conhecimento do problema apresentado pela criança e, conseqüentemente, para a orientação no âmbito da sua reabilitação.

Vejamos agora como é que estes indicadores (QIv, QIr, discrepâncias QIv-QIr) delimitam a caracterização cognitiva de vários grupos clínicos.

Assim, as crianças com espinha bifida e/ou hidrocefalia podem ter ụm padrão característico de funcionamento neuropsicológico que inclui dificuldades viso-motoras, compreensão reduzida da linguagem, problemas de memória e de atenção e QIv superior ao QIr (Wills, 1993; Baron et al., 1995 citados por Olds, Ju \& Dole, 1999)..O QIv é habitualmente mais elevado que o QIr nas crianças com hidrocefalia, podendo traduzir competências verbais superiores ao raciocínio abstrato e a aptidões visoperceptivas fracas (Holler, Fennel, Crosson, Boggs \& Mickle, 1995; Hurley, Dorman, Laatsch, Bell \&
D'Avignon, 1990). Alguns investigadores explicam estes déficits com base em lesões na substância branca do hemisfério direito. Mesmo nos casos de hidrocefalia moderada, o QI e o desempenho acadêmico situam-se aproximadamente um desvio-padrão abaixo da média da população normal (Hynd et al., 1998).

Por sua vez, a investigação de GangorosaEmerson, Jansiewicz, Emerson, Groover e Grant (1999) sugere que crianças com fenilcetonúria de Tipo IIA e de tipo IIB evidenciam um QIv significativamente mais reduzido comparativamente a um grupo de crianças com fenilcetonúria de tipo clássico.

Estudos de caso realizados por Fuld e Fisher (1977) sugerem que crianças com lesão cerebral traumática obtêm resultados mais baixos em testes de inteligência em comparação com estimativas do nível intelectual anterior à lesão. No entanto, e mais uma vez, existe alguma controvérsia no que diz respeito às discrepâncias entre QIv-QIr na WISC. Alguns estudos apontam para um padrão de resultados QIv < QIr; noutras pesquisas não foram encontradas diferenças (cf. Hynd et al., 1998). Tem sido sugerido que os QIr mais baixos podem resultar de déficits ao nível da percepção viso-espacial ou da rapidez de processamento (Dalby \& Obrutz, 1991).

As crianças com lesão cerebral traumática apresentam com frequiência diminuições mais acentuadas ou mais persistentes no QIr, do que no QIv, particularmente nos casos em que as lesões são severas (cf. Dalby \& Obrutz, 1991; Donders, 1997; Donders \& Warschausky, 1997). A capacidade de atenção, a memória, a aptidão motora ou a rapidez de processamento da informação encontram-se freqüentemente afetadas nestes casos. Daí que um ou mais déficits possam originar uma diminuição no desempenho dos subtestes que determinam o QIr.

Em contraponto aos QIr mais reduzidos nos casos de lesões severas é comum o registro de QIv mais elevados em crianças que foram vítimas de lesões no hemisfério esquerdo, numa idade mais prematura, antes dos cinco anos de idade (Cohen, Branch, Willis, Weyandt \& Hynd, 1992; Cohen, Branch, McKie \& Adams, 1994; Dalby \& Obrutz; 1991; Holler, Fennel, Crosson, Boggs \& Mickle, 1995). Por outro lado, as crianças que sofreram le- 


\section{Mário R. Simões}

sões no hemisfério direito, tendem a apresentar um abaixamento nos QIv e QIr (Woods, 1980). Woods interpreta esta evidência como sendo consistente com uma contribuição inicial do hemisfério esquerdo para as funções lingüísticas.

Como já foi referido, é necessária precaução na inferência de disfunção cerebral lateralizada, apenas com base nas discrepâncias QIv-QIr na WISC-III. Este tipo de inferência assenta na ideia simplista de que o hemisfério esquerdo regula primariamente as funções lingüísticas, ao mesmo tempo que o hemisfério direito se encontra envolvido essencialmente na mediação de tarefas viso-espaciais e não-verbais. É prematuro concluir que os indivíduos com lesões unilaterais que envolvem o hemisfério cerebral esquerdo devem apresentar discrepâncias entre QIv-QIr (QIr > QIv), ou que indivíduos com lesões unilaterais no hemisfério direito manifestam resultados de acordo com o padrão QIv > QIr. A literatura referente a adultos encontra uma base reduzida para esta noção e a investigação com a população pediátrica é ainda mais equívoca (cf. Hynd et al., 1998). Por exemplo, Cohen, Branch, McKie e Adams (1994) investigaram um grupo reduzido de crianças que experienciaram um único acidente vascular cerebral no hemisfério esquerdo ou direito. Os resultados indicaram que as crianças com dano no hemisfério esquerdo apresentam um declínio global nos testes de inteligência, com uma discrepância mínima entre o QIv-QIr a favor do QIr (4 pontos). As crianças com dano no hemisfério direito apresentaram um declínio acentuado apenas no QIr (discrepância de 13 pontos a favor do QIv).

Numa série de estudos, Jaffe \& cols. seguiram um grupo de crianças com lesão cerebral traumática. Apesar de terem ocorrido ganhos substanciais um ano depois, os resultados no QIr das crianças com lesões severas ou moderadas permaneceram mais baixos do que os do grupo de controlo. Três anos depois, as crianças e adolescentes com lesões moderadas ou severas ainda apresentavam déficits no funcionamento intelectual, raciocínio adaptativo, memória, aprendizagem conceptual e noutras aptidões (Jaffe, Polissar, Fay \& Liao, 1995). Estas descobertas confirmam a tendência da recuperação cognitiva ser inicialmente rápida e depois mais lenta. Num outro estudo sobre recuperação após lesão cerebral traumática em crianças escolarizadas, Yates e Taylor (1997) investigaram a alteração em várias medidas cognitivas incluindo os resultados obtidos no QIr. O grau de incremento do QIr (calculado a partir de uma Forma Reduzida da WISC-III que incluiu apenas os subtestes de Cubos e Composição de Objetos) estava relacionado com a severidade da lesão (medida pelos resultados na Glasgow Coma Scale e dados da neuroimagem). Neste âmbito, resta acrescentar que qualquer investigação sobre recuperação de desempenhos terá de contemplar questões inerentes às avaliações repetidas, como é o caso dos efeitos da prática. Estes têm sido observados na subescala de realização da WISC-III. Deste modo, os subtestes de realização apoiam-se até certo ponto no fator novidade.

\section{Índices Fatoriais}

Relativamente às versões anteriores, a WISCIII inclui um novo teste (Pesquisa de Símbolos) e, com base em diferentes agrupamentos de subtestes identificados a partir de técnicas de análise fatorial, oferece a possibilidade de calcular Indices Fatoriais. Assim, para além dos tradicionais resultados em QIs (QIEC, QIv e QIr), passam a ser calculados resultados para quatro Índices Fatoriais. Estes Indices são definidos do seguinte modo: Compreensão Verbal (baseado nos subtestes Informação, Semelhanças, Vocabulário e Compreensão); Organização Perceptiva (compreende o Completamento de Gravuras, Disposição de Gravuras, Cubos e Composição de Objetos); Resistência à Distração (composto pelos subtestes Aritmética e Memória de Dígitos) e Rapidez de Processamento (definido pelos subtestes Código e Pesquisa de Símbolos).

A validade do modelo fatorial com quatro Índices tem recebido apoio em estudos de replicação independentes com crianças normais (Roid, Prifitera, \& Weiss, 1993) e com lesão cerebral traumática (Donders \& Warschausky, 1996a). No entanto, outros investigadores têm questionado a validade da estrutura de quatro fatores. Little (1992) observa que a Memória de Dígitos tem uma saturação mínima no fator Resistência à Distração, sugerindo a supressão deste Indice. Por razões semelhantes, Sattler (1992) questiona a inclusão da Disposição de Gravuras no 
cálculo do fator Organização Perceptiva.

É importante examinar a validade e utilidade dos Índices Fatoriais na elaboração de hipóteses acerca do funcionamento neuropsicológico. À partida encontramos neste nível de análise relativo aos Índices Fatoriais uma possibilidade adicional de identificar padrões ou perfis de desempenho (áreas cognitivas fortes e fracas), presentes tanto em crianças "normais" como em crianças pertencentes a condições clínicas ou a grupos diagnósticos. É sugerido que os Índices Fatoriais poderão permitir um estudo mais preciso da vulnerabilidade seletiva e das taxas diferenciais de recuperação no domínio da inteligência. A interpretação a partir dos Indices Fatoriais poderá mesmo ser considerada preferível à análise dos padrões dos subtestes, uma vez que a fiabilidade é mais elevada nos resultados dos Índices Fatoriais. Como iremos ver de seguida, esta leitura parece ser mais legítima para os Índices Fatoriais constituídos por quatro subtestes (Compreensão Verbal e Organização Perceptiva) do que para aqueles que incluem apenas dois (f́ndices Resistência à Distração e Rapidez de Processamento).

A lesão cerebral traumática pediátrica pode causar déficits iniciais massivos, em várias áreas do funcionamento cognitivo. Neste contexto, Donders (1997) defende a possibilidade dos Indices Fatoriais permitirem discriminar os déficits viso-espaciais como causa primária dos resultados mais baixos no QIr da reduzida Rapidez de Processamento, nos casos de lesão cerebral.

Os resultados de várias investigações recentes sugerem um padrão comum de diminuição seletiva nos Índices Organização Perceptiva e Rapidez de Processamento e no QIr. Este padrão de resultados é raro na amostra de estandardizaçã̃o da WISCIII, mas freqüente após um episódio de lesão cerebral traumática grave (Donders, 1996a, 1997; Donders \& Warschausky, 1997). Os Índices Organização Perceptiva e Rapidez de Processamento apresentam ainda correlações estatisticamente significativas com variáveis neurológicas relacionadas com a localização e a gravidade da lesão (determinada pela duração do coma examinada a partir da Glasgow Coma Scale) e por dados provenientes de neuroimagem (Donders, 1997). O Índice Rapidez de Processamento é mesmo o resultado que melhor se correlaciona com a duração do coma em crianças com lesão cerebral traumática severa (Donders \& Warschausky, 1996a).

Os resultados sugerem que a Organização Perceptiva é tão sensível, quanto o QIr, às seqüelas da lesão cerebral traumática e que os déficits na Rapidez do Processamento de informação são bastante comuns após lesão cerebral pediátrica (Dennis, Wilkinson, Koski, \& Humphreys, 1995; Donders \& Warschausky, 1997). Ou seja, o Indice Rapidez de Processamento pode fornecer informação diagnióstica útil, no que diz respeito à capacidade da criança com lesão cerebral traumática processar informação com rapidez e eficiência. Este dado ilustra igualmente a importância da administração de rotina dos subtestes suplementares da WISC-III, como é especificamente o caso da Pesquisa de Símbolos.

O QIv e os Índices Compreensão Verbal ou Resistência à Distração não se relacionam nem com a gravidade da lesão, nem com a duração do coma (Donders, 1997), apoiando deste modo a idéia de que estes parâmetros não são sensíveis aos efeitos da lesão cerebral traumática. A idade no momento de ocorrência da lesão parece apresentar algum valor preditivo do resultado obtido no Índice Compreensão Verbal, uma vez que as crianças mais velhas alcançam melhores desempenhos que as crianças mais novas. O Índice Compreensão Verbal pode refletir aptidões completamente aprendidas que são com menor frequiuencia afetadas nos casos de lesão cerebral traumática. O Índice Resistência à Distração parece não constituir, nestas crianças, uma medida apropriada da atenção. No caso de crianças com lesão cerebral traumática a avaliação deverá ser complementada com medidas de atenção mais específicas (Donders, 1997). A idéia de que as crianças com lesão cerebral traumática apresentam déficits na atenção (e na rapidez de processamento) encontra-se documentada na literatura (Dalby \& Obrtuz, 1991; Fletcher et al., 1995).

Resultados semelhantes foram encontrados na pesquisa de Tremont, Wittner, Miller e Mittenberg (1997) junto de 33 crianças com traumatismo craniano: QIv (média $=85.9$ ) superior ao QIr (média $=78.45$ ); em $30 \%$ dos casos a diferença entre estes indicadores era significativa. No mesmo sentido foram identificadas discrepâncias entre os Indices Com- 
preensão Verbal e Organização Perceptiva e entre os Índices Resistência à Distração e Rapidez de Processamento. Foram ainda observadas relações significativas entre duração da amnésia pós-traumática e QIs $(r=-.34$ a -.44$)$ e entre resultados na Glasgow Coma Scale, o QIv e o QIEC $(r=.31)$. Neste estudo, a medida estandardizada da amnésia pós-traumática é o melhor preditor do QIEC medido após a lesão.

Num sentido parcialmente diferente encontram-se os resultados da investigação de Aubert e cols. (2001): o QIv, o QIEC e os Índices Compreensão Verbal e Rapidez de Processamento discriminam os desempenhos de 24 crianças com traumatismo craniano agudo dos resultados de crianças de um grupo de controlo.

Com o objetivo de verificar se as presentes conclusões se aplicam apenas a crianças com lesão cerebral traumática futuras investigações deverão examinar a estrutura fatorial e os subtipos de agrupamentos da WISC-III em grupos de crianças provenientes de grupos clínicos com outros tipos de disfunção cerebral.

De um modo geral, os Índices Fatoriais correlacionam-se com testes neuropsicológicos que medem constructos similares. Num estudo com 32 crianças candidatas à cirurgia da epilepsia, foram observadas relações entre déficits acadêmicos específicos (identificados a partir de provas estandardizadas de realização escolar) e padrões característicos na WISC-III e em outros testes neuropsicológicos. Neste sentido, os desempenhos reduzidos em testes de matemática estão associados a um padrão de resultados deficitários em tarefas de resolução de problemas e processamento não verbal (Índice Organização Perceptiva da WISC-III, Children's Category Test, Visuo-Motor Integration) e realizações pobres em testes de compreensão da leitura encontram-se ligados a déficits em tarefas de processamento verbal (Índice Compreensão Verbal da WISC-III, Boston Naming Test, California Verbal Learning Test-Children) (Craggs, Stanford \& Thornton, 1997).

Os resultados são contraditórios no que se refere ao Índice Resistência à Distração da WISCIII, também designado por Terceiro Fator. Uma pesquisa com 118 crianças e que recorreu a medidas auditivas e visuais do Test of Variables of Attention (TOVA) (Erros de Omissão, Erros de Intromissão, Tempo de Resposta, Variabilidade das Respostas, Respostas Antecipatórias, Respostas Múltiplas) sugere que o Índice. Resistência à Distração constitui uma medida de atenção e da memória de trabalho (Gliko, Escalona, Reinehr, Proctor-Weber \& Golden, 2001). Outra investigação com 426 criançàs encaminhadas para avaliação neuropsicológica, na sua maior parte com problemas neurológicos e/ou psicopatológicos, revela que o Índice Resistência à Distração da WISC-III não é uma medida válida da atenção uma vez que após o controlo de variáveis como a idade e os efeitos do nível intelectual aquele Índice não se correlaciona com medidas como o TOVA e várias escalas de avaliação de comportamentos completadas por pais e professores (Greene, Marsh, Katell, Proctor-Weber \& Golden, 2001).

Por outras palavras, e mais uma vez, os Índices Fatoriais não devem ser usados isolada ou exclusivamente para formular inferências clínicas de natureza diagnóstica. Vários estudos têm encontrado um apoio forte para os Índices Compreensão Verbal e Organização Perceptiva mas apenas uma evidência limitada para os Indices Resistência à Distração e Rapidez de Processamento (Kush, 1996). A investigação futura deverá fornecer linhas orientadoras adicionais para o uso e interpretação do terceiro e quarto fatores (Kamphaus, 1993; Kaufman, 1993) através do recurso a provas neuropsicológicas (Hynd et al., 1998). É possível que os resultados nalguns dos Índices da WISC-III sejam muito heterogêneos para que possam ser úteis no exame diagnóstico do funcionamento cognitivo.

\section{Análise por subteste}

E aconselhada a aplicação integral da totalidade dos subtestes da WISC-III. Todos os subtestes devem ser valorizados do ponto de vista da avaliação, incluindo os que são considerados como facultativos e não entram no cálculo dos três quocientes de base (QIv, QIr, QIEC). Ao contrário da análise item a item, onde a apreciação qualitativa poderá ser de grande importância, a observaçăo do perfil constituído pelas pontuações ponderadas de cada subescala e de cada subteste comporta uma explica- 
ção de natureza quantitativa. Os desempenhos da criança em cada subteste são, ao mesmo tempo, diretamente comparados com os resultados nos restantes subtestes e com os do seu grupo etário, colocando assim em evidência áreas fortes e fracas. O reagrupamento de alguns subtestes, no interior de cada subescala, pode também revelar aspectos importantes para a explicação do funcionamento cognitivo da criança.

Consideremos alguns exemplos de agrupamentos de subtestes que viabilizam uma análise de perfis de resultados obtidos. Assim, a atenção e as memórias auditiva, de trabalho, imediata, ou a longo termo são fortemente solicitadas sempre que as pontuações padronizadas dos subtestes Informação, Aritmética e Memória de Dígitos apresentam alguma homogeneidade. Resultados fracos nestas provas são observados, com freqüência, em crianças desatentas-impulsivas.

Muitas vezes, as crianças desatentas-impulsivas obtêm igualmente resultados mais baixos nos subtestes Código, Pesquisa de Símbolos e Labirintos.

As competências de integração lingüística e de expressão do pensamento podem também ser postas à prova pelos subtestes de Semelhanças, Vocabulário e Compreensão.

As competências lexicais são examinadas a partir do Vocabulário e da Informação.

Por outro lado, existe uma estreita ligação entre os subtestes Cubos e Composição de Objetos que examinam o raciocínio não verbal e viso-espacial. $O$ insucesso nestas provas constitui, muitas vezes, um índice de desorganização viso-espacial.

O fracasso limitado apenas à Disposição de Gravuras pode estar associado a uma fragilidade das competências lingüísticas.

$\mathrm{O}$ exame das funções motoras ou práxicas pode ser considerado a partir da observação das respostas em tarefas motoras, como é o caso dos Labirintos ou do Código.

Os subtestes Composição de Objetos, Labirintos e Cubos são potencialmente úteis na avaliação neuropsicológica da dispraxia.

Num outro registo, Fennell (2000) sugere a presença de vários subtestes da WISC-III numa Bateria Abreviada de Avaliação Neuropsicológica da Criança, com o objetivo de examinar as seguintes funções: Cognição (Vocabulário, Compreensão, Cubos e Composição de Objetos), Memória (Memória de Dígitos), Funções Frontais (Pesquisa de Símbolos e Labirintos). Por sua vez, Middleton (2001) seleciona numa bateria de avaliação neuropsicológica algumas provas da WISC-III destinadas ao exame de determinadas funçōes: Código e Pesquisa de Símbolos (Rapidez do Processamento de informação); QIv (Linguagem e comunicação); QIr (Percepção visual); QIEC (Competência intelectual geral).

Retomando trabalhos de vários investigadores (Goia, Isquith \& Guy, 2001, pp. 329-336; Grégoire, 2000, pp. 176-204; Kaufman \& Lichtenberger, 2000, 81-190; Lussier \& Flessas, 2001, pp. 51-58) é possível sublinhar análises relativas à interpretação associadas aos subtestes considerados isoladamente.

\section{Sub-escala Verbal}

Informação. Mede o nível dos conhecimentos adquiridos a partir da educação na escola e na família. Apela à memória episódica a longo termo. Permite verificar a organização temporal. Nas crianças que apresentam problemas de linguagem (disfasias), dificuldades de aprendizagem (déficits seqüenciais) ou desatenção-impulsividade é particularmente freqüente a existência de um déficit na organização temporal. Proporciona também uma idéia acerca da curiosidade intelectual dos sujeitos mais velhos em relação às ciências.

Semelhanças. Examina a capacidade de estabelecer relações lógicas e a formação de conceitos verbais ou de categorias. Avalia a capacidade de sintese e de integração de conhecimentos. É um subteste difícil para as crianças com limitações intelectuais. Pode ser o melhor resultado da subescala verbal para os sujeitos disfásicos que freqüentemente apresentam um nível elevado de inteligência geral e, em especial, uma boa capacidade de síntese. Do ponto de vista da avaliação das funções executivas é importante observar se a criança alcança a pontuação máxima nos itens através de uma única resposta correta ou de explicações pormenorizadas.

Aritmética. Avalia a capacidade de cálculo mental, a compreensão de enunciados verbais de uma certa 


\section{Mário R. Simōes}

complexidade e a capacidade de raciocinio. E bastante sensível a um déficit de atenção (e à falta de controlo da impulsividade). Requer uma boa capacidade da memória de trabalho (e da memória para seqüências de procedimentos) necessária para manter presente todos os elementos do problema a resolver. $\mathrm{O}$ examinador deverá estar atento ao modo de resolıção adaptado pela criança, sobretudo quando a sua resposta está errada.

Vocabulário. Mede a competência lingüística, os conhecimentos lexicais e, sobretudo, a facilidade de elaboração do discurso. Tal como no subteste das Semelhanças, é de observar a justeza do vocabulário utilizado e a precisão do pensamento. Bernstein (2001) lembra que constitui uma simplificação grosseira considerar que o resultado nesta prova faculta alguma informação relativa à integridade do hemisfério esquerdo. Um desempenho baixo pode traduzir falta de familiarização com o contexto educativo ou ausência de experiência escolar. Um resultado elevado nesta prova de conhecimento do vocabulário (freqüentemente objeto de uma aprendizagem intensa) não constitui uma garantia de que o hemisfério esquerdo se encontra intacto.

Compreensão. Examina a capacidade do sujeito exprimir as suas experiências. Apela ao conhecimento de regras de relacionamento social. Permite observar quer a facilidade de argumentação (quando é pedido ao sujeito para justificar as suas respostas), quer a flexibilidade mental (quando é solicitada uma segunda resposta ao mesmo item). Um resultado fraco pode sugerir uma certa forma de inércia frontal (nos sujeitos que experimentam dificuldades neurológicas na mobilização dos seus recursos cognitivos durante a tentativa de evocação de vários soluções para um mesmo problema) ou revelar desconhecimento das regras sociais, falta de empatia e de julgamento (que caracterizam freqüentemente os sujeitos que apresentam uma disfunção não verbal).

Memória de Dígitos. A distinção das tarefas nesta prova, tradicionalmente conceptualizada como sendo de memória verbal, é defendida por investigadores como Riccio e Reynolds (1998) que contestam a habitual combinação dos resultados da Ordem Dire- ta (atenção) e da Ordem Inversa (inteligência geral). Em termos globais a prova de Memória de Dígitos está associada também ao processamento verbal auditivo (Pospisil, Selden, Michaels, DevarajuBackhaus \& Golden, 2001). A Memória de Dígitos na Ordem Directa mede a memória auditiva seqüencial e é bastante sensível à capacidade de escuta e às flutuações da atenção. Quando o sujeito repete todos os números, mas não na ordem em que eles the foram apresentados, trata-se especificamente de capacidade de evocação seqüencial em modalidade auditiva e não de um déficit de natureza mnésica ou atencional. A Memória de Dígitos no Sentido Inverso mede a capacidade de memória de trabalho. Esta tarefa é geralmente mais difícil que a precedente. É esperado que o resultado na Ordem Inversa seja um ou dois pontos inferiores ao obtido na Ordem Direta. Um resultado (excepcional) igual ou superior na Ordem Inversa parece indicativo do recurso a excelentes estratégias executivas e da utilização preferencial de um modo de evocação visual (que substitui uma atenção auditiva enfraquecida).

\section{Sub-Escala de Realização}

Completamente de Gravuras. É o primeiro subteste da escala a ser aplicado podendo, por isso, esperarse que o resultado obtido seja negativamente influenciado pelo efeito de novidade, sobretudo numa criança tímida, por uma reação de inibição ansiosa. Apela a uma forma de memória visual e a um bom senso prático. $\mathrm{O}$ examinador deve pedir ao sujeito, sobretudo aos mais velhos (10 anos ou mais), para nomear a parte que falta, uma vez que a resposta pode proporcionar um bom índice das suas capacidades de acesso lexical (escolha da palavra exata) e da sua cultura geral. As crianças impulsivas, bem como os sujeitos disfásicos, freqüentemente apresentam uma tendência para utilizar termos vagos, em vez de evocar o termo exato. À semelhança do subteste Informação, uma fraca escolarização, ou a pertença a meio sócio-cultural desfavorecido, pode explicar a pobreza do vocabulário utilizado pelo sujeito.

Código. Mede a capacidade de associar números a símbolos e de memorizar corretamente essas associações, a fim de executar a tarefa o mais rapidamente 
possível. Avalia a capacidade de aprendizagem «mecânica», automatizada. Um bom resultado sugere um estilo sequiencial preferencial. A reprodução dos símbolos requer uma boa caligrafia, muitas vezes ausente nas crianças impulsivas (os problemas neuromotores finos são freqüientemente relacionados com esta problemática). Um resultado fraco pode depender de uma dificuldade da memória cinestésica da sequiência gestual a executar, ou ser observado em certas crianças dispráxicas.

Disposição de Gravuras. Requer uma boa capacidade de análise perceptiva, bem como uma integração do conjunto das informações disponíveis. Uma pontuação fraca pode refletir um dano nas funções frontais de auto-regulação. A relação dos desenhos que compõem cada história exige uma forma de discurso interior que pode manifestar-se não funcional nos sujeitos que apresentam uma disfasia, sobretudo receptiva. Muitas vezes as crianças disfásicas evidenciam dificuldades na percepção do tempo e do espaço, que podem ser detectadas nesta tarefa.

Cubos. Examina a capacidade de organização e processamento viso-espacial/não-verbal, a capacidade para decompor mentalmente os elementos constituintes do modelo a reproduzir. É considerada uma medida de resolução de problemas não verbais $\mathrm{e}$ usada como uma das contra-provas de déficits nas funçōes executivas. Neste contexto, permite identificar dificuldades de auto-monitorizaçāo presentes quando a criança é incapaz de reconhecer erros evidentes, mesmo quando é desafiada a descobrir esses erros ou a comparar o seu trabalho com o estímulo, após a aplicação formal do subteste. A escolha do tipo de estratégia (global, analítica ou sintética) que permite a execução da țarefa revela-se um excelente índice da inteligência não-verbal, bem como das capacidades de raciocinio viso-espacial. Dificuldades na manipulação do material e, sobretudo, na organização espacial dos elementos, podem dificultar o desempenho neste subteste nos casos de dispraxia de construção (e inteligência normal). Em comparação com outras medidas de aptidão viso-espacial, o subteste de Cubos supõe o recurso a um funcionamento viso-perceptivo, capacidades construtivas, coordenação, rapidez psicomotora.
Composição de Objetos. Mede a capacidade de organizar um todo a partir de elementos separados, apela à capacidade de integração perceptiva é sensível à dispraxia de construção. Proporciona uma oportunidade para observar diretamente a estratégia de resolução dos problemas (itens).

Pesquisa de Símbolos. Apela para a capacidade de discriminação perceptiva. Depende de uma boa capacidade de atenção visual e de memória de trabalho. Carone e cols. (2000) defendem que este subteste pode substituir não apenas o Código, como refere por exemplo o manual americano da WISC-III, mas qualquer outro subteste de realização, sem que essa alteração possa distorcer os resultados QIr ou QIEC no exame de crianças com traumatismo craniano. As crianças impulsivas, ou com déficit de atenção, obtêm com freqüência os resultados mais baixos, da subescala de realização, no Código e na Pesquisa de Símbolos. Quando o Código é melhor sucedido que a Pesquisa de Símbolos, prevalece a suspeita de uma dificuldade de ordem grafo-motora, associada à execução de símbolos inabituais, permanecendo totalmente preservadas as competências perceptivas. Se a Pesquisa de Símbolos é uma prova que apresenta resultados superiores relativamente ao Código, trata-se de uma criança que não aprendeu a auto-regular o seu desempenho numa tarefa cronometrada, mais do que um déficit de atenção visual e/ou da discriminação perceptiva, ou da existência de um controlo insuficiente da impulsividade.

Labirintos. Examina a capacidade de antecipação e de planificação, requer uma estratégia viso-espacial em memória de trabalho. É bastante sensível à impulsividade do método ou abordagem adaptada. As crianças mais jovens, que sofrem de dispraxia motora, apresentam dificuldades na realização desta tarefa: o insucesso provém de uma incapacidade de planificação da sua execução gestual, como se existisse uma desconexão entre a intenção e a realização do gesto a efetuar.

\section{Variabilidade inter-testes}

Uma dispersão acentuada das pontuações nos vários subtestes da WISC-III pode ter interesse clínico e introduzir informação adicional importante 
para o exame neuropsicológico. Manga e Fournier (1997) referem que há tendência para suspeitar da existência de alguma alteração, neurológica ou outra, sempre que a variabilidade inter-testes é elevada (p. ex., pontos de corte superiores ao percentil 93 ou ao percentil 98 ).

\section{Formas Reduzidas}

O tempo disponível para a avaliação neuropsicológica pode constituir um problema, tanto mais que a aplicação da totalidade dos subtestes da WISC-III pode exigir mais do que uma hora. A utilização de Formas Reduzidas da WISC-III tem sido sugerida sempre que a totalidade dos subtestes da escala não pode ser integralmente aplicada, devido a constrangimentos temporais ou à fadiga do sujeito. Por outro lado, alguns destes subtestes não são sensíveis a uma diminuição do funcionamento cerebral, quando comparados com medidas neuropsicológicas específicas (Fletcher et al., 1995).

O número de testes que constitui estas Formas Reduzidas oscila freqüentemente entre dois subtestes (Vocabulário e Cubos), quatro subtestes (Semelhanças, Vocabulário, Disposição de Gravuras, Cubos) ou mesmo seis subtestes (todos os atrás referidos). A partir de uma amostra de 568 crianças encaminhadas para avaliação neuropsicológica Frazen e Smith-Seemiller (2001) verificaram que o recurso a Formas Reduzidas constituídas por seis testes não introduz vantagens adicionais relativamente ao uso de formas abreviadas que incluem dois subtestes ou quatro subtestes: as correlações com o QIEC são relativamente elevadas, ainda que variáveis ( .87 a .97) em comparação com as correlações entre 6 testes e o $\operatorname{QIEC~(.93~a~.95).~}$

Uma Forma Reduzida da WISC-III, constituída pelos subtestes de Informação, Vocabulário, Completamento de Gravuras, Cubos e Código (coeficientes de correlação de .94 entre QI da forma abreviada e o QIEC), pode constituir uma alternativa razoável para avaliar as capacidades cognitivas de crianças diagnosticadas quer com distúrbio de hiperatividade com déficit de atenção (Furgueson, McGuffin, Greenstein \& Soffer, 1998), quer com perturbações da conduta e perturbações de oposição (Furgueson, Greenstein, McGuffin \& Soffler, 1999).
Donders e Warschausky (1996b) comprovaram a validade de uma outra Forma Reduzida da WISC-III, numa amostra de crianças com lesão cerebral traumática, que permite o cálculo de estimativas quer do QIEC, quer dos quatro fndices Fatoriais. Esta Forma incluiu as Semelhanças e o Vocabulário (como medidas da Compreensão Verbal), Completamento de Gravuras e Cubos (como medidas de Organização Perceptiva), Aritmética e Memória de Dígitos (como medidas de Resistência à Distração), e Código e Pesquisa de Símbolos (como medidas de Rapidez de Processamento). Destes oito subtestes, seis foram escolhidos para a construção de um Índice da Escala Completa desta Forma Abreviada. Excluiram-se os subtestes Memória de Dígitos e Pesquisa de Símbolos, uma vez que normalmente não são considerados para o cálculo do QIEC da WISC-III. Esta Forma Reduzida permite ao psicólogo economizar mais de um quarto do tempo de administração e, ainda assim, obter a mesma informação, em termos de resultados nos Índices Fatoriais. As análises realizadas utilizando a amostra de estandardização americana da WISC-III apontam para um nível de fiabilidade bastante aceitável para esta Forma Reduzida. Os resultados desta investigação também apresentam para um nível de validade aceitável.

É necessário proceder a validações adicionais desta Forma Reduzida noutros grupos clínicos. Além disso, esta Forma Reduzida não deve ser utilizada em contextos de tomada de decisão, sobretudo quando é elevada a dispersão de resultados padronizados entre os subtestes da Compreensão Verbal e/ou entre os subtestes da Organização Perceptiva. Nestas circunstâncias, é desejável a administração dos restantes subtestes de cada uma das subescalas, bem como o cálculo usual dos resultados nos Índices.

\section{Avaliação do nível de funcionamento cognitivo pré-mórbido}

Compreender o nivel de funcionamento cognitivo pré-mórbido constitui uma área de particular interesse na avaliação neuropsicológica. A maior parte das tentativas para estimar o estatuto prémórbido dizem respeito à inteligência psicométrica. Do ponto de vista da avaliação é importante reco- 
nhecer que muitas crianças com lesão cerebral moderada evidenciam problemas cognitivos e comportamentais cujo aparecimento é anterior à ocorrência da lesão. Por isso, o conhecimento do funcionamento cognitivo prévio da criança é essencial para compreender as conseqüências e quantificar as mudanças na aptidão global ocorridas após uma lesão cerebral ou uma doença neurológica (epilepsia, tumor cerebral, meningite, etc.).

A estimativa da inteligência pré-mórbida nas crianças arrasta uma série dificuldades específicas adicionais, que resultam do fato das suas aptidões não estarem completamente desenvolvidas e de com freqüência elas não alcançarem níveis de funcionamento estáveis antes do início da lesão ou da doença. Tais fatos explicam o relativo fracasso das tentativas para desenvolver medidas de competência prémórbida para crianças (Yates \& Taylor, 1997).

Utilizando os dados de estandardização americana da WISC-III, Vanderploeg, Schinka, Baum, Tremont e Mittenberg (1998) desenvolveram dois métodos para estimar a competência pré-mórbida nas crianças. Um deles consistiu em estimar o QI a partir de variáveis demográficas, constituindo a média dos anos de escolarização dos pais e o grupo étnico da criança os melhores preditores. No outro método de estimativa da inteligência pré-mórbida foram utilizadas estas variáveis demográficas e o melhor desempenho nos subtestes de Vocabulário ou de Completamento de Gravuras. Os dois subtestes da WISC-III foram escolhidos pela sua adaptabilidade à lesão (recuperação rápida dos desempenhos). A utilidade potencial destes subtestes, como estimativa do nível pré-mórbido de funcionamento cognitivo, diminui com o tempo.

Outros investigadores têm sugerido a utilização da informação relativa à inteligência dos pais ou irmãos, como um índice da competência esperada nas crianças (Baron, 2001; Boll \& Stanford, 1997; Redfield, 2001; Reynolds, 1997a, 1997b). Nos casos de exposição de crianças a determinadas substâncias químicas como o chumbo; a informação acerca dos QIs dos pais ou irmãos é frequientemente utilizada pelos advogados de defesa sendo comparada com o QI da criança (Mealey's National Lead Litigation Conference, 1999 citado em Redfield, 2001). A inteligência encontra-se correlacionada sig- nificativamente entre os membros da mesma família, mas a um nível que limita a fiabilidade das estimativas do QI de uma criança com base em medidas de competência de outros membros da família.

\section{Testes neuropsicológicos e funções psicológicas examinadas a partir da WISC-III}

Alguns estudos recorrem a resultados na WISC-III como critério de validação de provas (neuro)psicológicas. Outros estudos examinam ain$\mathrm{da}$, em diferentes grupos clínicos, as relações entre resultados na WISC-III e testes neuropsicológicos considerados como tendo fundamentos sólidos. Estes dois aspectos, a seguir abordados, permitem compreender melhor as relações entre constructos e o significado dos desempenhos na WISC-III.

Neste plano, a investigação sugere que as medidas de funções executivas são relativamente independentes do desempenho de testes de inteligência. A partir de uma amostra de 421 crianças encaminhadas para avaliação neuropsicológica, com perturbações neurológicas e/ou psicopatológicas, Reinehr, Mleko, Escalona, Borosh e Golden (2001) constataram a existência de relações estatisticamente significativas, quase sempre de sentido negativo $(-0.19$ a -0.42$)$, entre os desempenhos nos vários indicadores que é possível obter a partir da WISC-III e em provas reputadas de avaliação de funções executivas (Wisconsin Card Sorting Test, Trail Making Test $A$ e B).

Outros estudos sugerem a existência de uma relação significativa entre inteligência (examinada a partir da WISC-III) e a memória (avaliada com o Test of Memory and Learning) em alunos com dificuldades de aprendizagem (Raggio, D'Amato \& Hoerig, 1999).

A partir de um grupo de 330 crianças encaminhadas para avaliação neuropsicológica observouse que a inteligência e a memória, respectivamente avaliadas através da WISC-III e do Wide Range Assessment Memory and Learning (WRAML), constituem competências cognitivas fortemente interdependentes (van den Broek, Sellers, Golden, Burns \& Drabman, 2001). Outros estudos que apontam para a ausência de correlações entre a WISC-III e outras provas neuropsicológicas (Wide Range 
Assessment Memory and Learning, Continuous Performance Test) colocam em questão muitas estratégias interpretativas (van den BroeK, Mattis \& Golden, 1998). Neste contexto, Chandler, Burns, Sellers, Golden e Willen (2001) referem a superioridade da WISC-III (em comparação com o WRAML) na diferenciação de grupos clínicos (hiperactividade com défice de atençãoldificuldades de aprendizagem) relativamente a grupos controlo (existência de um funcionamento intelectual superior ao funcionamento mnésico) na deteç̧ão de déficits de processamento.

Por sua vez, o Peabody Picture Vocabulary Test-Revised (PPVT-R) pode constituir um instrumento de despistagem apropriado do QIv e Índice Compreensão Verbal da WISC-III em crianças com traumatismo craniano (Natale et al., 2000).

Com base nos resultados da WISC-III, WISCR e Halstead-Reitan Neuropsychological Battery, Gray, Livingston, Marshall, Jennings e Haak (1999) sugerem a possibilidade das crianças em idade escolar, encaminhadas para avaliação, manifestarem principalmente diferenças de nível mais do que no padrão de funcionamento neuropsicológico.

As relações entre constructos (inteligência, memória, linguagem, atenção, etc.) têm sido objeto de alguma investigação que é necessário continuar recorrendo à WISC-III e a outras provas de referência que habitualmente fazem parte da avaliação neuropsicológica. Uma melhor compreensão das relações entre estas variáveis (neuro)psicológicas constitui um apoio decisivo para o trabalho de diagnóstico e prognóstico. É este o contexto de um nosso projeto de investigação, presentemente em curso e que pretende comparar os resultados obtidos em diferentes instrumentos de avaliação que examinam funções cognitivas distintas (WISC-III, provas neuropsicológicas de memória, linguagem, atenção, funções executivas, etc.) em grupos normativos e em grupos clínicos. Aperfeiçoar a base conceptual de provas neuropsicológicas, viabilizada pela consideração do nível intelectual (e de vários outros parâmetros interpretativos) e pelas várias possibilidades de comparação entre diferentes testes neuropsicológicos, é um dos objetivos essenciais deste projeto. Outro objetivo inclui a elaboração de dados normativos relativos aos desempenhos em testes neuropsicológicos por parte de crianças e ado- lescentes pertencentes a grupos sem dificuldades ou com problemas neurologicos bem definidos.

\section{Investigações com outros grupos clínicos}

A esmagadora maioria das investigações recenseadas incluem crianças com idades compreendidas entre os 6 e os 12 anos, encaminhadas para situações de avaliação neuropsicológica. Nalguns casos a natureza reduzida das amostras inviabiliza a generalização dos resultados.

Alguns dos indicadores que é possível obter a partir da WISC-III (QIv, QIr, QIEC, Indice Compreensão Verbal, subtestes de Vocabulário e Composição de Objetos) discriminam os desempenhos de 24 crianças com diagnóstico psiquiátrico dos resultados de 21 crianças sem diagnóstico que faziam parte de um grupo de controlo. Os autores desta investigação (Natale, Burns, Golden, Escalona, Mleko \& Valley-Grey, 2001) explicam estas discrepâncias como consequiência dos efeitos cumulativos dos problemas psicopatológicos no desenvolvimento intelectual.

Epilepsia. A criança com epilepsia apresenta com freqüência um nível mais baixo de funcionamento intelectual ("normal fraco", "zona limite") no que se refere ao QIv, QIr, QIEC, bem como aos Índices Fatoriais (Cohen, Branch, Riccio \& Hall, 1991). Para uma melhor compreensão das relações entre epilepsia e inteligência é necessário considerar variáveis como a idade de aparecimento das crises (o risco de déficit intelectual é maior quando as convulsões têm início na primeira infância), o tipo de epilepsia, os efeitos isolados e combinados das medicações antiepiléticas. Neste contexto, a WISC-III pode ser usada também na despistagem das funções específicas (memória, atenção, etc.) mais afetadas por aquelas variáveis.

Cancro. A avaliação neuropsicológica e o recurso à WISC-III servem para identificar sequielas e declínios progressivos e específicos no padrão de funcionamento cognitivo secundários ao cancro no SNC, ou em resultado dos tratamentos radiológicos: por exemplo, dificuldade de aquisição de informação nova (cf. Hill \& Rath, 1998; Palmer, Glass, Redddick, Goloubeva, Gajjar, Merchant \& Mulhern, 2000). HIV. O QIEC determinado a partir do recurso a uma 
Forma Reduzida WISC-III discrimina não apenas o desempenho de 9 crianças com HIV de 11 crianças com traumatismo craniano moderado ou com distúrbio de hiperatividade com déficit de atençāo, diferenciando igualmente os desempenhos das crianças com HIV na WISC-III relativamente a provas de memória e aprendizagem como o WRAML (no HIV - QI é mais reduzido do que os resultados naquela prova de memória e aprendizagem) (Simpson, Patton, Widmayer, Peterson, Starratt, Burns \& Kehoe, 1999). Um outro estudo evidencia a existência de correlações estatisticamente significativas entre número de células D-4 (que constitui um indicador da progressão da doença) e os Índices Rapidez do Processamento e Resistência à Distração (Askinazi, Grant, Legido \& Bagarazzi, 2000).

Síndroma de Gilles de la Tourette. As crianças com este problema têm geralmente um rendimento intelectual na WISC-III semelhante às crianças do seu grupo etário. Os resultados no QIr são significativamente inferiores, especialmente devido ao desempenho nos subtestes com tempo limite. Ainda que obtenham sucesso no mesmo número de itens as crianças necessitam de mais tempo para responder, o que sugere a existência de uma lentidão ideativa (e não um problema de raciocínio). As dificuldades nas tarefas de planificação podem ser ilustradas a partir dos erros de planificação nos Labirintos. Lussier e Flessas (2001) referem a presença de uma disfunção frontal nas crianças com este diagnóstico.

\section{Testar os limites}

Existem vários tipos de materiais complementares que reforçam o uso e a interpretação dos resultados com a WISC-III. Um exemplo recente, neste contexto, é o da WISC-III PI ("WISC-III como Instrumento de Avaliação de Processos", Kaplan, Fein, Morris, Kramer \& Delis, 1999) que corresponde a um conjunto de procedimentos desenvolvidos com o objetivo de assegurar uma compreensão mais completa acerca das razões (ou processos) subjacentes a um resultado particular.

Tendo em vista esta compreensão das dificuldades, pode ser desejável testar-os-limites (testingthe-limits) durante ou após a aplicação da totalidade da prova. Para os subtestes Disposição de Gravuras,
Cubos, Composição de Objetos, Labirintos, pode permitir-se que a criança termine a sua tarefa depois do tempo limite, caso ela manifeste esse desejo. Vários resultados fora dos limites de tempo permitidos, apontam para uma lentidão no tratamento da informação, de natureza perceptiva ou ideativa, ou ainda por uma lentidão na execução associada a um problema motor. Por vezes, é desejável que se aplique a prova para lá do número consentido de erros consecutivos, verificando assim se o sujeito consegue resolver outros itens com um nível de dificuldade superior. Neste caso, o fracasso em itens fáceis é freqüentemente considerado como indicador de um déficit de atenção ou ainda de uma extraordinária lentidão na adaptação à tarefa, que sugere ansiedade ou rigidez cognitiva. Assim, Lussier e Flessas (2001) propõem o cálculo de novas cotações ponderadas, que permitiram uma melhor apreciação das áreas fortes e fracas da criança sem constrangimentos de tempo. Poderá ser proposta à criança qualquer outra adaptação na apresentação de um item, que possa ser útil e facilite a emissão de uma resposta.

\section{Futuro próximo}

O papel da avaliação neuropsicológica centrado na localização cerebral diminuiu substancialmente com o advento da neuroimagem e de outras técnicas diagnósticas atualmente disponíveis (Tomografia Computadorizada, Ressonância Magnética, Tomografia por Emissão de Positrões, Tomografia Computadorizada por Emissão de Fotões). Estas permitem uma circunscrição mais precisa das lesões cerebrais. A elucidação das relações cérebro-comportamento passa pois, de forma decisiva, pelo recurso a estas novas tecnologias de visualização do cérebro. Neste contexto, é de esperar um melhor conhecimento das variáveis neuropsicológicas colocadas em jogo na resposta aos vários subtestes e indicadores proporcionados pela WISC-III. Existem já alguns dados que ilustram o fato de que alguns processos e funções avaliados pela WISC-III poderão ter alguma associação teoricamente consistente com sistemas neurológicos (Hynd, Cohen, Riccio \& Arcenaux, 1998). A pesquisa exploratória de Patton, Selevan-Eisenstein, Burns, Montgomery, Simco e Widmayer (2000) procura 
quantificar as relações entre a atividade alfa, identificada a partir da electroencefalografia quantitativa, e a inteligência. Alguma evidência experimental apoia igualmente a ligação entre localização anatômica e desempenhos de crianças normais no Indice Compreensão Verbal (Morgan, Hynd, Hall, Novey \& Eliopulos, 1997 citados em Hynd \& cols., 1998). A investigação futura poderá facultar mais informações acerca da localização das funções e processos mentais avaliados nos vários subtestes da WISC-III. Até lá a WISC-III continuará a proporcionar informação de grande utilidade que é necessário interpretar, nomeadamente, no contexto dos dados provenientes da utilização de uma bateria (apropriada) de testes neuropsicológicos.

\section{Referências Bibliograficas}

Anderson, V., Northam, E., Hendy, J. \& Wrennall, J. (2001). Developmental neuropsychology: $A$ clinical approach. East Sussex: Psychology Press.

Anderson, V. \& Gilandis, A. (1994). Neuropsychological assessment of learning disabilities. In S. Touyez, D. Byrne \& A. Gilandis (Eds.), Neuropsychology in clinical practice (pp. 128-161). New York: Academic Press.

Askinazi, L.A., Grant, M.L., Legido, A., \& Bagarazzi, M.L. (2000). The relationship between performance on neuropsychological test measures and immunologic and virologic markers of disease progression in HIV infected children. Archives of Clinical Neuropsychology, 15, 801-802.

Aubert, M.J., Chandler, M., Willen, E., Burns, W.J., Golden, C.J., Widmayer, S., Peterson, L., Starratt, C. \& Puranik, S. (2001). Memory, verbal comprehension, and processing speed discriminate children with TBI. Archives of Clinical Neuropsychology, 16, 772.

Baron, I.S. (2001). Clinical implications and practical applications of child neuropsychological evaluations. In K.O. Yeates, M.D. Ris \& H.G. Taylor (Eds.), Pediatric neuropsychology: Research, theory, and practice (pp. 439-456). New York: Guilford.
Bernstein, J.H. (2001). Developmental neuropsychological assessment. In K. O. Yates, M. D. Ris \& H. G. Taylor (Eds.), Pediatric neuropsychology: Research, theory, and practice (pp. 405-438). New York: Guilford.

Boll, T.J. \& Stanford, L.D. (1997). Pediatric brain injury. In C.R. Reynolds \& E. Flecher-Janzen (Eds.), Handbook of clinical child neuropsychology ( $2^{\text {nd }}$ ed., pp. 140-156). New York: Plenum.

Carone Jr, D., Patton, D., Bums, W. J., Starrat, C., Natale, M., Simpson, R., Bradshaw, A., Widmayer, S., Peterson, L. \& Puranik, S. (2000). Pediatric closed head injury: Using Symbol Search as a substitute on the WISC-III. Archives of Clinical Neuropsychology, 15, 795.

Chandler, M., Burns, J., Sellers, A., Golden, C J. \& Willen, E. (2001). Distributional differences between ADHD/LD and controls WISC-III and WRAML index discrepancies. Archives of Clinical Neuropsychology, 16, 774-775.

Cohen, M.J., Branch, W.B., McKie, V.C. \& Adams, R. J. (1994). Neuropsychological impairment in children with sickle cell anemia and cerebrovascular accidents. Clinic Pediatrics, 517-524.

Cohen, M.J., Branch, W.B., Riccio, C.A. \& Hall, L.L. (1991). Intellectual functioning of children with epilepsy: Comparison of performance on the WISC-III and WISC-R. Paper presented at the $99^{\text {th }}$ Annual Convention of the American Psychological Association, San Francisco.

Cohen, M.J., Branch, W.B., Willis, W.G., Weyandt, L.L. \& Hynd, G.W. (1992). Childhood. In A. E. Puente \& R. J. McCaffrey (Eds.), Handbook of neuropsychological assessment (pp. 49-79). New York: Plenum.

Craggs, J.C., Stanford, L.D. \& Thornton, A.E. (1997). Neuropsychological functioning and seizure focus: Relationship to academic achievement in children with epilepsy. Archives of Clinical Neuropsychology, 12, 303.

Dalby, P.R. \& Obrutz, J.E. (1991). Epidemiologic characteristics and sequelae of closed headinjured children and adolescents: A review. 
Developmental Neuropsychology, 7, 35-68.

Dennis, M., Wilkinson, M., Koski, L. \& Humphreys, R.P. (1995). Attention deficits in the long term after childhood head injury. In S. H. Broman \& M. E. Michel (Eds.), Traumatic head injury in children (pp. 165-187). New York: Oxford University Press.

Donders, J. (1997). Sensitivity of the WISC-III to injury severity in children with traumatic head injury. Assessment, 4, 107-109.

Donders, J. \& Warschausky, S. (1996a). A structural equation analysis of the WISC-III in children with traumatic head injury. Child Neuropsychology, 2, 185-192.

Donders, J. \& Warschausky, S. (1996b). Validity of a short form of the WISC-III in children with traumatic head injury. Child Neuropsychology, 2, 227-232.

Donders, J. \& Warschausky, S. (1997). WISC-III factor index score patterns after traumatic head injury in children. Child Neuropsychology, 3, 7178.

Fennell, E.B. (2000). Issues in child neuropsychological assessment. In R.D. Vanderploeg (Ed.), Clinician's guide to neuropsychological assessment $\left(2^{\text {nd }} \mathrm{ed} ., \mathrm{pp} .357\right.$ 381). Mahwah: Erlbaum.

Figueiredo, V.L.M. (2000). WISC-III. In J.A. Cunha e cols. (Eds.), Psicodiagnóstico - V (5.2 ed. rev. e ampliada, pp. 603-614). Porto Alegre: Artmed Editora.

Fletcher, J.M., Levin, H.S. \& Butler, I.J. (1995). Neurobehavioral effects of brain injury on children: Hydrocephalus, traumatic brain injury, and cerebral palsy. In M.C. Roberts (Ed.), Handbook of pediatric psychology (pp. 362-383). New York: Guilford.

Franzen, M.D. \& Smith-Seemiller, L. (2001). Comparison among seven different short forms of the WISC-III. Archives of Clinical Neuropsychology, 16, 825.

Franzen, M.D. (2000). Reliability and validity in neuropsychological assessment ( $2^{\text {nd }}$ ed.). New York: Kluwer/Plenum.
Fuld, P.A. \& Fisher, P. (1977). Recovery of intelectual ability after closed head injury. Developmental Medicine and Child Neurology, 19, 495-502.

Furgueson, C., Greenstein, D., McGuffin, P. \& Soffer, S. (1999). Efficacy of the WISC-III short form for children diagnosed with conduct and oppositional defiant disorders. Archives of Clinical Neuropsychology, 14, 6-7.

Furgueson, C., McGuffin, P., Greenstein, D. \& Soffer, S. (1998). Efficacy of the WISC-III short form for children diagnosed with ADHD. Archives of Clinical Neuropsychology, 13, 10.

Gangorosa-Emerson, M.E., Jansiewicz, E.M., Emerson, R.L., Groover, W.D. \& Grant, M.L. (1999). Cognitive differences in children with Type IIA and Classical PKU. Archives of Clinical Neuropsychology, 14, 7.

Gioia, G.A., Isquith, P.K. \& Guy, S.C. (2001). Assessment of executive functions in children with neurological impairment. In R.J. Simeonsson \& S. R. Rosenthal (Eds.), Psychological and developmental assessment: Children with disabilities and chronic conditions (pp. 317-356). New York: Guilford.

Gliko, B., Escalona, A.M., Reinehr, J., ProctorWeber, Z., Golden, C.J. (2001). Correlations between attentional measures and measures of learning in children with ADHD. Archives of Clinical Neuropsychology, 16, 778.

Gray, R., Livingston, R., Marshall, R., Jennings, E. \& Haak, R. (1999). Empirically derived neuropsychological subgroups of school-age children. Archives of Clinical Neuropsychology, 14,8 .

Greene, L. R., Marsh J., Katell, M., Proctor-Weber, Z. \& Golden, C. J. (2001). Relationships among neuropsychological measures of attention and freedom from distractibility. Archives of Clinical Neuropsychology, 16, 778-779.

Grégoire, J. (2000). L'évaluation clinique de l'intelligence de l'enfant: Théorie et pratique $d u$ WISC-III. Liège : Pierre Mardaga.

Groth-Marnat, G., Gallagher, R.E., Hale, J.B. \& 
Kaplan, E. (2000). The Wechsler Intelligence Scales. In G. Groth-Marnat (Ed.), Neuropsychological assessment in clinical practice: A guide to test interpretation and integration (pp. 129-194). New York: Wiley.

Hill, J.M., \& Rath, J F. (1998). The effect of neuropsychological deficits in childhood cancer on parental functioning. Archives of Clinical Neuropsychology, 13,13.

Holler, K.A., Fennell, E.B., Crosson, B., Boggs, S.R. \& Mickle, J.P. (1995). Neuropsychological and adaptative functioning in younger versus older shunted for early hydrocephalus. Child Neuropsychology, I, 63-73.

Horowitz, T., Schatz, P. \& Chute, D. (1997). Trends in neuropsychological test usage. Archives of Clinical Neuropsychology, 12, 338-339.

Humphries, T. \& Bone, J. (1993). Use of IQ criteria for evaluating the uniqueness of the learning disability profile. Journal of Learning Disabilities, 26, 348-351.

Hurley, A.D., Dorman, C., Laatsch, L., Bell, S. \& D'Avignon, J. (1990). Cognitive functioning in patients with spina bifida, hydrocephalus, and the "cocktail party" syndrome. Developmental Neuropsychology, 6, 151-172.

Hynd, G.W., Cohen, M.J., Riccio, C.A. \& Arceneaux, J.M. (1998). Neuropsychological basis of intelligence and the WISC-III. In A. Prifitera \& D.H. Saklofske (Eds.),WISC-III clinical use and interpretation: Scientist-practioner perspectives (pp. 203-226). New York: Academic Press.

Jaffe, K.M., Polissar, N.L., Fay, G.C. \& Liào, S. (1995). Recovery trends over three years following pediatric traumatic brain injury. Archives of Physical Medicine and Rehabilitation, 76, 17-26.

Kamphaus, R.W. (1993). Clinical assessment of children's intelligence. Needham Heights: Allyn \& Bacon.

Kaplan, E., Fein, D., Kramer, J. \& Delis, D. (1999). WISC-III PI: Manual. San Antonio: The Psychological Corporation.

Kaufman, A.S. (1993). King WISC the third assu- mes the throne. Journal of School Psychology, $31,345-354$.

Kaufman, A.S. (1994). Intelligence testing with WISC-III. New York: Wiley.

Kaufman, A.S. \& Lichtenberger, E.O. (2000). Essentials of WISC-III and WPPSI-R. New York: Wiley.

Kay, J., \& Warschausky, S. (1999). WISC-III index growth curve characteristics following traumatic brain injury. Journal of Clinical and Experimental Neuropsychology, 21(2), 186-199.

Kush, J.C. (1996). Factor structure of the WISC-III for students with learning disabilities. Journal of Psychoeducational Assessment, 14, 32-40.

Lezak, M.D. (1988). IQ: RIP. Journal of Clinical and Experimental Neuropsychology, 10, 351-361.

Lezak, M.D. (1995). Neuropsychological assessment $\left(3^{\text {rd }}\right.$ ed.). New York : Oxford University Press.

Little, S.G. (1992). The WISC-III: Everything old is new again. School $P$ sychology $Q$ uarterly, 7, 148154.

Lussier, F. \& Flessas, J. (2001). Neuropsychologie de l'enfant: Troubles dévelopmentaux et de l'apprentissage. Paris: Dunod.

Manga, D., \& Fournier, C. (1997). Neuropsicología clínica infantil: Estudio de casos en edad escolar. Madrid: Editorial Universitas.

Middleton, J.A. (2001). Practioner review: Psychological sequelae of head injury in children and adolescents. Journal of Child Psychology and Psychiatry, 42 (2), 165-180.

Moffitt, T.E. \& Silva, P.A. (1987). WISC-R verbal and performance IQ discrepancies in an unselected cohort: Clinical significance and longitudinal stability. Journal of Consulting and Clinical Psychology, 55, 768-774.

Natale, M.J., Burns, W. J., Golden, C.J., Escalona, A.M., Mleko, A. \& Valley-Grey, S. (2001). The psychiatric diagnosis: WISC-III relationship revisited. Archives of Clinical Neuropsychology, 16, 794-795.

Natale, M.J., Patton, D., Burns, W.J., Carone, D., Simpson, R., Bradshaw, A., Widmayer, S., 
Puranick, S., Peterson, L. \& Starratt C. (2000). Receptive language performance as an estimator of verbal intellectual performance in children with head injuries. Archives of Clinical Neuropsychology, 15, 808.

Nielsen, K.H., Hynd, G.W. \& Hiemenz, J.R. (1998). Planum temporale assymetry and VIQ $>$ PIQ/ PIQ>VIQ splits on the WISC-III. Archives of Clinical Neuropsychology, 13, 87.

Olds, J., Ju, J., \& Doyle, D. (1999). Mathematical abilities in adolescents with spina bifida and hydrocephalus. Archives of Clinical Neuropsychology, 14, 14-15.

Palmer, S.L., Glass, J.O., Reddick, W.E., Goloubeva, O., Gajjar, A., Merchant, T.E. \& Mulhern, R.K. (2000). Patterns of cognitive functioning among survivors of pediatric medulloblastoma: A longitudinal analysis. Archives of Clinical Neuropsychology, 15, 792.

Patton, D.E., Selevan-Eisenstein, E., Burns, W.J., Montgomery, D., Simco, E.R. \& Widmayer, S. (2000). QEEG asymmetry ratio is related to intelligence. Archives of Clinical Neuropsychology, 15, 799.

Phelps, L. Leguori, S., Nisewaner, K. \& Parker, M. (1993). Practical interpretation of the WISC-III with language disordered children. Journal of Psychoeducational Assessment (WISC-III Monograph), 71-76.

Pospisil, T., Selden, J., Michaels, D., DevarajuBackhaus, S. \& Golden, C.J. (2001). Factor analysis of attentional tests in ADHD. Archives of Clinical Neuropsychology, 16, 783.

Prifitera, A. \& Dersh, J. (1993). Base rates of WISCIII diagnostic subtest patterns among normal, learning disabled, and ADHD samples. Journal of Psychoeducational Assessment (WISC-III Monograph), 43-55.

Raggio, D.J., D'Amato, R. \& Hoerig, D. (1999). Comparing the TOMAL, CPT, and WISC-III: Are memory, attention and intelligence related? Archives of Clinical Neuropsychology, 14, 16.

Redfield, J. (2001). Familial intelligence as na estimate of expected ability in children. Clinical
Neuropsychologist, 15(4), 446-460.

Reinehr, J., Mleko, A.L., Escalona, A.M., Borosh, B. \& Golden, C.J. (2001). Intelligence and executive function in a pediatric neuropsychiatric population. Archives of Clinical Neuropsychology, 16, 783.

Reynolds, C.R. (1997a). Measurement and statistical problems in neuropsychological assessment of children. In C. R. Reynolds \& E. Flecther-Janzen (Eds.), Handbook of clinical child neuropsychology ( $2^{\text {nd }}$ ed., pp. 180-203). New York: Plenum.

Reynolds, C.R. (1997b). Postscripts on premorbid ability estimation: Conceptual addenda and a few words on alternative and conditional approaches. Archives of Clinical Neuropsychology, 12, 769778.

Riccio, C.A. \& Reynolds, C.R. (1998). Neuropsychological assessment of children. In A.S. Bellack \& M. Hersen (Eds.), Comprehensive clinical psychology (Vol. 4: Assessment, pp. 267-301). Oxford: Elsevier.

Riva, D. \& Cazzaniga, L. (1986). Late effects of unilateral brain lesions sustained before and after age one. Neuropsicologia, 24, 423-428.

Roid, G.H., Prifitera, A. \& Weiss, L.G. (1993). Factor struture in an independent sample. Journal of Psychoeducational Assessment (WISC-III Monograph), 6-21.

Saling, M. (1994). Neuropsychology beyond 2000: The failure of diagnostic expertise. In S. Touyez, D. Byrne \& A. Gilandis (Eds.), Neuropsychology in clinical practice (pp. 3-14). New York: Academic Press.

Sattler, J.M. (1992). Assessment of children. (revised and update $3^{\text {rd }}$ ed.). San Diego: Author

Simeonsson, R. \& Rosenthal, S. (2001). Psychological and developmental assessment: Children with disabilities and chronic conditions. New York: Guilford.

Simpson, R.A., Patton, D.E., Widmayer, S., Peterson, L., Starratt, C., Burns, W.J., \& Kehoe, F. (1999). Intelligence and memory: A comparison between pediatric TBI, HIV and ADHD patients. Archives 
132 Mário R. Simões

of Clinical Neuropsychology, 14, 19.

Slate, J.R. (1995). Discrepancies between IQ and index scores for a clinical sample of students: Useful diagnostic indicators? Psychology in the Schools, 32, 103-108.

Spreen, O. \& Strauss, E. (1998). A compendium of neuropsychological tests $\left(2^{\text {nd }} \mathrm{ed}\right.$.). New York: Oxford University Press.

Telzrow, C.F. (1989). Neuropsychological applications of common educational and psychological tests. In C.R. Reynolds \& E. Flecther-Janzen (Eds.), Handbook of clinical child neuropsychology (pp. 357-376). New York: Plenum.

Tremont, G., Wittner, M.S., Miller, L.J., \& Mittenberg, W. (1997). WISC-III performance following pediatric head trauma. Archives of Clinical Neuropsychology, 12, 418-419.

van den Broek, A. L., Sellers, A., Golden, C.J., Burns, W.J. \& Drabman, R . (2001). The multidimensional relationship of the WISC-III and WRAML. Archives of Clinical Neuropsychology, 16, 771 .

van den Broek, A., Mattis, K. \& Golden, C.J. (1998). Intelligence, memory, and attention in children: A correlational study. Archives of Clinical Neuropsychology, 13, 87.

Vanderploeg, R.D., Schinka, J.A., Baum, K.M., Tremont, G., \& Mittenberg, W. (1998). WISCIII premorbid prediction strategies: Demographic and best performance approaches. Psychological Assessment, 10, 277-284.

Walsh, K.W. (1994). Neuropsychology: A clinical approach ( $3^{\text {rd }}$ ed.). New York: Churchill Livingstone.

Wechsler, D. (1991). Wechsler Intelligence Scale for Children - Third Edition (WISC-III): Manual. San Antonio: The Psychological Corporation

Woods, B.T. (1980). The restricted effects of right hemisphere lesions after age one: Wechsler test data. Neuropsychologia, 18, 65-70.

Yates, K.O. \& Taylor, H.G. (1997). Predicting premorbid neuropsychological functioning following pediatric traumatic brain injury.

\section{Journal of Clinical and Experimental} Neuropsychology, 19, 825-837.

Este trabalho insere-se no contexto de um projeto de investigação financiado pela Fundação para a Ciência e a Tecnologia (FCT): "Aferição de testes neuropsicológicos para a população portuguesa". A redação deste texto beneficiou igualmente do apoio do Centro de Psicopedagogia da Universidade de Coimbra. 